\title{
Hippocampal Gamma Oscillations Increase with Memory Load
}

\author{
Marieke K. van Vugt, ${ }^{1}$ Andreas Schulze-Bonhage, ${ }^{2}$ Brian Litt, ${ }^{3,4}$ Armin Brandt, ${ }^{2}$ and Michael J. Kahana ${ }^{5}$ \\ ${ }^{1}$ Princeton Neuroscience Institute, Princeton University, Princeton, New Jersey 08540, ${ }^{2}$ University Hospital of Freiburg, D-79106 Freiburg, Germany, \\ and Departments of ${ }^{3}$ Neurology, ${ }^{4}$ Bioengineering, and ${ }^{5}$ Psychology, University of Pennsylvania, Philadelphia, Pennsylvania 19104
}

\begin{abstract}
Although the hippocampus plays a crucial role in encoding and retrieval of contextually mediated episodic memories, considerable controversy surrounds the role of the hippocampus in short-term or working memory. To examine both hippocampal and neocortical contributions to working memory function, we recorded electrocorticographic activity from widespread cortical and subcortical sites as 20 neurosurgical patients performed working memory tasks. These recordings revealed significant increases in $48-90 \mathrm{~Hz}$ gamma oscillatory power with memory load for two classes of stimuli: letters and faces. Sites exhibiting gamma increases with memory load appeared primarily in the hippocampus and medial temporal lobe. These findings implicate gamma oscillatory activity in the maintenance of both letters and faces in working memory and provide the first direct evidence for modulation of hippocampal gamma oscillations as humans perform a working memory task.
\end{abstract}

\section{Introduction}

Working memory refers to the capacity to maintain multiple item representations over a brief retention interval. This form of memory has been posited to be functionally and neurally distinct from episodic memory, which refers to the ability to remember experiences as being embedded in a unique temporal context (Tulving, 1983; Postle, 2006). Whereas episodic memory depends on the hippocampus and medial temporal lobe (MTL) structures, working memory is thought to be largely mediated by the prefrontal cortex (PFC) (Curtis and D'Esposito, 2003) with little or no hippocampal involvement (Baddeley and Warrington, 1970; Squire et al., 1993). This dissociation between working memory and episodic memory has been challenged, however, by recent findings that hippocampal activity is modulated during working memory tasks, and prefrontal activity is modulated during episodic memory tasks (Ranganath, 2006; Polyn and Kahana, 2008).

To assess the neural correlates of working memory function, researchers manipulate the number of items being held in mind - the memory load_- during a memory maintenance interval between study and test. Measures of brain activity that significantly correlate with memory load are seen as reflecting the neural processes involved in memory maintenance.

Received Feb. 3, 2009; revised Nov. 3, 2009; accepted Dec. 29, 2009.

We acknowledge support from the National Science Foundation Grant SBE 0354378 (Center of Excellence for Learning in Education, Science, and Technology), National Institute of Mental Health Grant P50 MH062196 (Conte (enter), and National Institutes of Health Grants R01 MH061975, MH068404, and MH055678. This research was also supported by the Deutsche Forschungsgemeinschaft (Sonderforschungsbereich 780, Synaptic Mechanisms, TPC3). We thank Josh Jacobs, Jeremy Manning, Sean Polyn, and Christoph Weidemann for helpful discussions.

This article is freely available online through the J Neurosci Open Choice option.

Correspondence should be addressed to Marieke K. van Vugt, Princeton Neuroscience Institute, Green Hall, Princeton University, Princeton, New Jersey 08540. E-mail: mkvan@princeton.edu.

DOI:10.1523/JNEUROSCI.0567-09.2010

Copyright $\odot 2010$ the authors $\quad 0270-6474 / 10 / 302694-06 \$ 15.00 / 0$
Although various neural measures have been shown to correlate with memory load, our focus here is on measures of oscillatory activity in the local field potentials recorded from subdural and depth electrodes in neurosurgical patients. Such recordings often reveal striking oscillatory effects, especially in the $4-9 \mathrm{~Hz}$ theta and $28-100 \mathrm{~Hz}$ gamma frequency bands, and activity in these bands has been implicated in a wide range of memory and cognitive processes (for review, see Kahana, 2006).

Several studies of working memory have found increases in gamma activity with memory load (Howard et al., 2003; Axmacher et al., 2007; Meltzer et al., 2008). Using letters as stimuli, Howard et al. (2003) found increases in gamma power with memory load at frontal and temporal recording electrodes in two neurosurgical patients. Using digits as stimuli and recording from a larger sample of patients, Meltzer et al. (2008) found increases in gamma power with memory load at widespread cortical regions. Using faces as stimuli, Axmacher et al. (2007) found increases in gamma power with memory load in the MTL. Their gamma effect was significant in the rhinal cortex, but not statistically reliable in the hippocampus. In addition to load-related changes in cortical gamma oscillations, cortical theta oscillations have also been found to correlate with memory load, but the direction of those correlations is not consistent across brain regions, stimulus materials, or even participants (Jensen and Tesche, 2002; Howard et al., 2003; Meltzer et al., 2008; Michels et al., 2008).

None of the studies cited above show load-dependent oscillatory activity in the hippocampus, an issue that is of crucial importance to the debate over the involvement of the hippocampus in working memory. A related question is whether hippocampal involvement differs depending on the nature and verbalizability of the stimuli. To address these questions, we analyzed a large intracranial EEG (iEEG) dataset collected during working memory tasks for two classes of stimuli: faces and letters. We devel- 


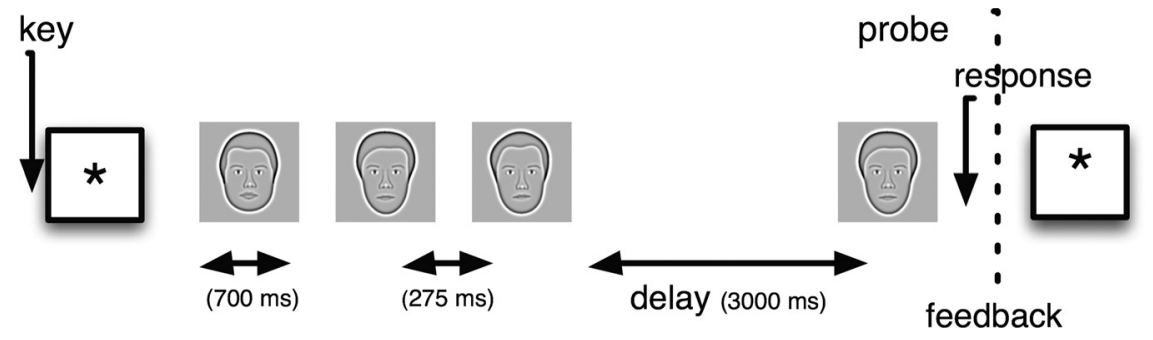

Figure 1. Schematic of the Sternberg task. Participants see a list of 1-4 stimuli (faces or letters), followed by a 3000 ms delay interval. After the delay, a probe stimulus appears and participants have to indicate whether the probe was a member of the just-presented list. The maintenance interval is defined as the period between 0 and $3000 \mathrm{~ms}$ after the offset of the last list item. Stimuli were sets of 16 letters and 16 faces, presented in alternating blocks of 15 lists.

oped region-of-interest (ROI) analyses to assess the regional specificity of oscillatory effects in key regions that have been previously implicated in episodic memory and working memory functions.

\section{Materials and Methods}

Twenty patients $(12$ male, mean age $=32, \mathrm{SD}=13)$ participated in a Sternberg (1966) short-term recognition memory paradigm involving lists of synthetic faces (Fig. 1) or the consonants of the Roman alphabet (henceforth letters). We conducted the study while patients were being monitored with subdural electrocorticographic (ECoG) and/or depth electrodes to localize seizure onset and for functional mapping. Patients were enrolled at the epilepsy monitoring units at the Hospital of the University of Pennsylvania in Philadelphia, PA, Brigham and Women's Hospital in Boston, MA, and the Universitäts Klinikum in Freiburg, Germany. Our research protocol was approved by the Institutional Review Boards at these institutions, and informed consent was obtained from all participants. Electrode placements were determined solely on the basis of clinical considerations. The 20 participants contributed signals from a total of 1454 electrodes distributed across varied brain regions (see Fig. 3). We retained for subsequent analyses 554 of these recordings because they satisfied several inclusion criteria, as described below.

Figure 1 illustrates the sequence of events during each trial of the experiment. Following the appearance of a fixation stimulus (an asterisk that appeared for 1000-1075 ms, jittered) on the display of a laptop computer, participants viewed a short series of letters or faces. Each stimulus appeared for $700-775 \mathrm{~ms}$, followed by a $275-350 \mathrm{~ms}$ interstimulus interval. After a $3000-3300 \mathrm{~ms}$ retention interval, a probe item appeared and participants indicated with a key press whether the probe was a member of the just presented series (a target) or an item not shown on the current list (a lure). After each trial, participants were given accuracy feedback. The participant advanced to the next trial with a key press. Temporal jitter was used to avoid spurious correlations between ongoing oscillations and the structure of the task. The experiment was programmed in the Python Experimental Programming Library (PyEPL) (Geller et al., 2007).

Lists comprised one, two, three, or four letters or one, two or three faces (fewer faces were used because accuracy drops off quickly when going from three to four faces), and the face and letter trials were presented in separate blocks of 15 trials. Study items were constrained such that none could be repeated on successive lists. Lists were constructed so that the frequencies of targets and lures and each of the list lengths were matched. In addition, targets were equally likely to match a study item from each serial position. Every session was preceded by two 16-trial training blocks plus 40 additional one-item lists to familiarize the participant with the face stimuli. Participants were given feedback on their average accuracy and reaction time (RT) at the end of each block. Incorrect trials and trials with RTs shorter than $200 \mathrm{~ms}$ or longer than $3500 \mathrm{~ms}$ were removed from the analysis.

Rather than using photographs of faces, we adopted a set of synthetic faces derived from real photographs but filtered to remove components like hair and skin texture while preserving the shape, size, and position of key facial features (Wilson et al., 2002). These faces are nonetheless realistic enough to generate strong responses in the fusiform face area (Loffler et al., 2005). Detailed information concerning the specific set of faces used in the present study are provided in Pantelis et al. (2008).

Electrocorticographic and depth electrode recordings. The local field potential was amplified and digitally recorded at sampling rates between 250 and $1024 \mathrm{~Hz}$ and bandpass filtered between 0.1 and $100 \mathrm{~Hz}$. Data were subsequently notch filtered with a Butterworth filter with zero phase distortion between 48 and 52 $\mathrm{Hz}$ (Freiburg recordings) or 58 and $62 \mathrm{~Hz}$ to eliminate the relevant line noise. Electrodes overlying epileptogenic regions, as indicated by the attending neurologists, were discarded. In addition, the intervals of interest were scanned for epileptic spikes and sharp waves by means of a kurtosis threshold (see supplemental Fig. 1, available at www.jneurosci.org as supplemental material); events were discarded if their kurtosis exceeded a threshold of 5 (Delorme and Makeig, 2004; Robinson et al., 2004; Sederberg et al., 2007a).

To synchronize electrophysiological recordings with behavioral events, the laptop computer generating the task sent pulses through the parallel or universal serial bus port via an optical isolator into an unused recording channel or digital input on the amplifier to time stamp the digital iEEG recording. The time stamps associated with these pulses aligned the experimental computer's clock with the iEEG clock to a precision well under the sampling interval of the iEEG recording $(<4 \mathrm{~ms})$. For all participants, the locations of implanted electrodes were determined by means of coregistered postoperative computed tomographies and preoperative magnetic resonance imaging (MRI) or from postoperative MRIs by an indirect stereotactic technique and converted into MNI (Montreal Neurological Institute) coordinates. Localization of depth electrode contacts in the hippocampus was done manually through clinicians' inspection of the postoperative MRIs.

Data analysis. Oscillatory power was computed using six-cycle Morlet wavelets (Schiff et al., 1994) at logarithmically spaced frequencies $\left(2^{x / 4}\right.$ $\mathrm{Hz}$ for $x \in 4, \ldots, 48)$. Wavelets are currently the standard method for quantifying oscillatory brain activity. See van Vugt et al. (2007) for a detailed comparison of oscillation detection methods, including wavelets, multitapers, and $P_{\text {episode. }}$. We converted absolute power into $z$-scores normalized by the mean and SD of the power during each particular trial's fixation interval. We then combined oscillatory power in seven frequency bands (delta, 2-4 Hz; theta, 4-9 Hz; alpha, 9-14 Hz; beta, $14-28 \mathrm{~Hz}$; low gamma, $28-48 \mathrm{~Hz}$; mid-gamma, $48-90 \mathrm{~Hz}$; high gamma, 90-100 Hz). During the maintenance interval, we computed mean power between 750 and $3000 \mathrm{~ms}$ after onset of the last study item (to avoid stimulus-related processing in the first $750 \mathrm{~ms}$ after stimulus onset).

We then regressed each trial's list length on the $z$-transformed oscillatory power for each channel and frequency and determined the $t$-statistic and $p$-value for these regression coefficients. For every regression, a corresponding permutation distribution with 1000 iterations was computed by randomly reassigning each oscillatory power value (dependent variable) to each list length (independent variable) and recomputing the regression coefficients. This created a distribution of regression coefficients corresponding to the null hypothesis that there is no relation between oscillatory power and memory load. Within each frequency band, we then took the regression coefficient with the minimum $p$-value for the subsequent analysis. Finally, we compared the empirical to the permutation distributions of $p$-values at the corresponding frequency.

All our analyses were done in regions of interest that have been previously associated with memory and attention. For individual electrode analyses, we counted the number of electrodes in each region exhibiting statistically significant positive or negative effects. Significance was compared to a threshold of $p=0.01$. To compare numbers of significant 
electrodes across ROIs and stimulus types, we used a Mantel-Haenszel test. This test is more appropriate than a $\chi^{2}$ test when comparing counts based on (occasionally) small numbers of observations.

To determine whether any ROI showed an aggregate effect, we adapted methods developed by Sederberg et al. (2007b). For each participant, we aggregated across all electrodes in each region of interest by taking the mean of the inverse normal transformed $p$-values (thereby turning them into $z$-values), and the same was done for the permutation distribution. We then combined across participants by summing the thus obtained $z$-values for both the empirical data and the permutation distribution. The significance of each ROI and frequency combination was determined by finding where each empirical summed- $z$-value fell within the permutation distribution. In other words, summed- $z$-values larger than all permuted summed- $z$-values would have a very small $p$-value. The resulting $p$-values were corrected for multiple comparisons using a false discovery rate (FDR) threshold of 0.005 . This significance threshold indicates that only $0.5 \%$ of the significant ROIs across all frequency bands and time intervals will be false positives. This differs from conventional $p$-value testing, where a $p$-value of 0.05 indicates that $\sim 5 \%$ of the effects that reach the significance threshold can be false positives.

To investigate whether any of the significant effects changed over time, we computed the summed- $z$-values in three equal time bins spanning the $3 \mathrm{~s}$ maintenance interval. We then compared the SD over time of these empirical sum- $z$-values to the SDs of the corresponding permutation distributions.

\section{Results}

Participants' mean accuracy was $92.1 \%$ for letters and $67.2 \%$ for faces. Their mean RT for correct trials was $1283 \mathrm{~ms}$ for letters and $1742 \mathrm{~ms}$ for faces. The finding of superior accuracy and shorter response times for letters than for faces replicates several previous studies (Hwang et al., 2005; Jacobs et al., 2006; van Vugt et al., 2009).

We first asked whether oscillatory activity during the maintenance interval varied with memory load across individual electrodes in four brain ROIs: dorsolateral PFC (DLPFC; Brodmann areas 9, 10 , and 46), temporal cortex (TC; Brodmann areas 20, 21, and 22), MTL (Brodmann areas 28,35 , and 36 , but excluding hippocampus), and hippocampus proper. Table 1 reports the number of participants and electrodes contributing data to each of these ROIs.

At each recording site, we correlated $z$-transformed oscillatory power with memory load in seven frequency bands as follows: delta, 2-4 Hz; theta, 4-9; alpha, 9-14 $\mathrm{Hz}$; beta, 14-28 Hz; low gamma, 28-48 Hz; mid-gamma, 48-90 $\mathrm{Hz}$; and high gamma, 90-100 Hz. We designated recordings that achieved a statistically significant correlation $(p<0.01)$ as showing a positive or negative memory load effect.

Figure 2 shows the proportions of electrodes exhibiting positive and negative memory load effects for each frequency band and ROI. Although individual electrodes showed both significant
Table 1. Number of participants and electrodes contributing data to each ROI

\begin{tabular}{lccccc}
\hline & Left & & \multicolumn{2}{l}{ Right } \\
\cline { 2 - 3 } \cline { 5 - 6 } Region & Participants & Electrodes & & Participants & Electrodes \\
\hline DLPFC & 6 & 25 & & 7 & 62 \\
Temporal & 10 & 129 & & 13 & 212 \\
MTL $^{a}$ & 7 & 40 & & 10 & 33 \\
Hippocampal $^{n}$ & 6 & 20 & & 9 & 33 \\
\hline
\end{tabular}

Data reflect the number of electrodes analyzed after excluding electrodes overlying epileptogenic regions (see Materials and Methods for details on exclusion criteria).

${ }^{a}$ Excluding hippocampus.

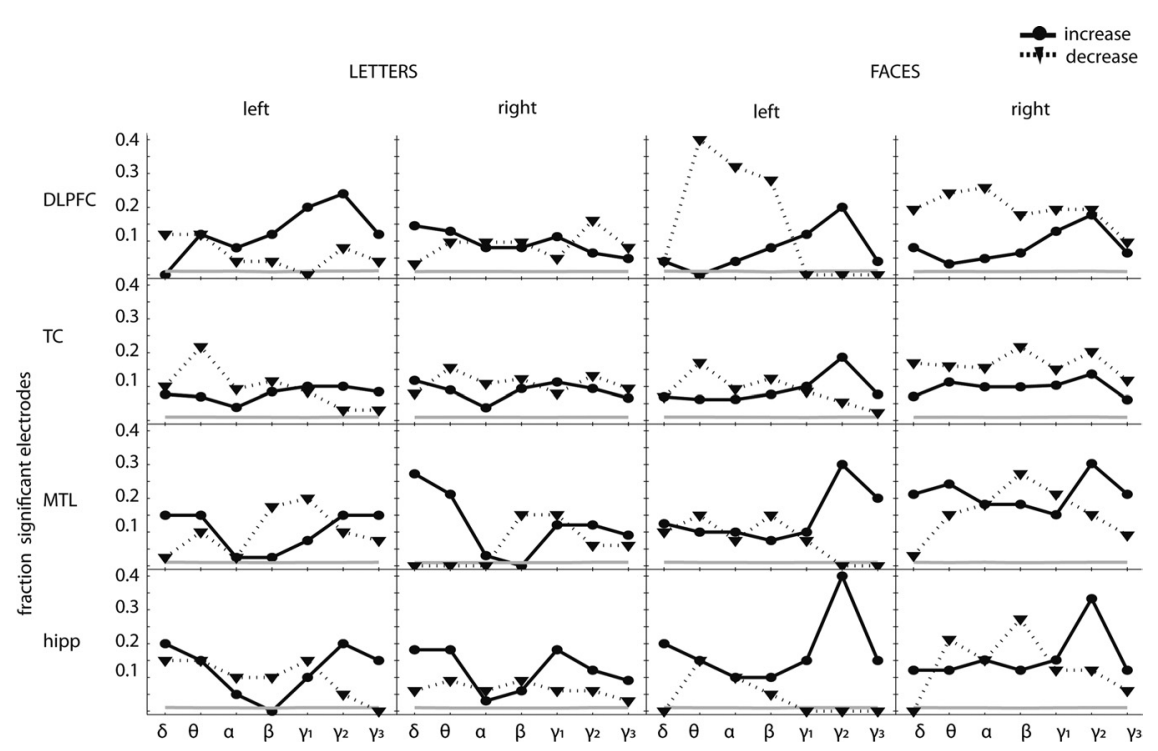

Figure 2. Single electrode analyses by region of interest during the maintenance interval. Fraction of electrodes showing a significant increase (solid) or decrease (dotted) of oscillatory power with memory load. The gray line indicates the fraction of significant electrodes in the permuted data. The frequency bands are as follows: delta $(\delta), 2-4 \mathrm{~Hz}$; theta $(\theta), 4-9 \mathrm{~Hz}$; alpha $(\alpha)$, 9-14 Hz; beta $(\beta), 14-28 \mathrm{~Hz}$; low gamma $\left(\gamma_{1}\right), 28-48 \mathrm{~Hz}$; mid-gamma $\left(\gamma_{2}\right), 48-90 \mathrm{~Hz}$; high gamma $\left(\gamma_{3}\right), 90-100 \mathrm{~Hz}$.

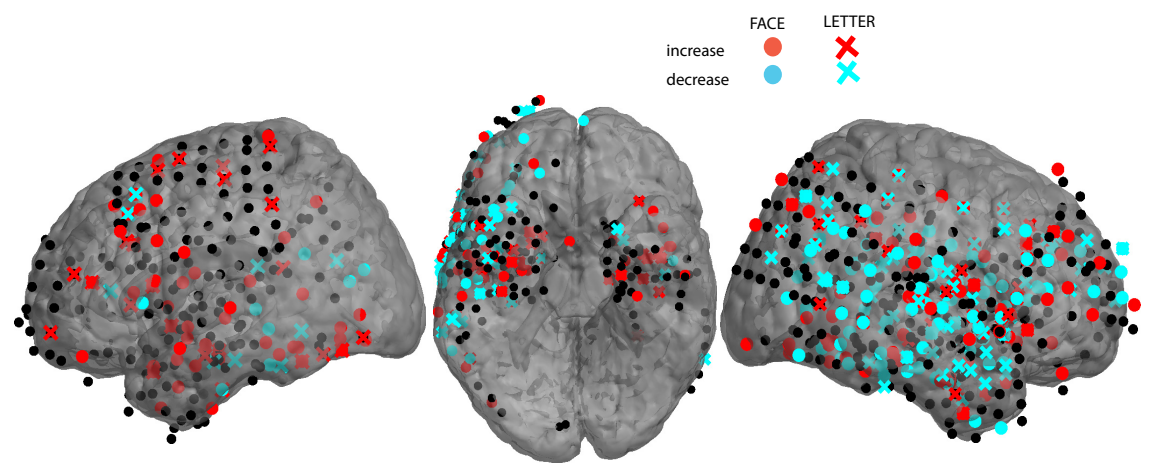

Figure 3. Cortical electrodes showing significant increases (red) and decreases (blue) in $48-90 \mathrm{~Hz}$ gamma power with memory load. Circles, Faces; crosses, letters. Electrodes not showing a significant load effect in this frequency band are drawn in black. Hippocampal electrodes are not shown. positive and negative load effects in various frequency bands, several trends may be seen. First, in the mid-gamma frequency band, the proportions of positive memory load effects exceeded that of negative memory load effects in the hippocampus $(0.25 \mathrm{vs}$ 0.07 ) and in the MTL ( 0.22 vs 0.08$)$. Collapsing across left and right ROIs and stimulus type, both of these differences were statistically significant [Mantel-Haenszel test values $(\mathrm{MH})=3.4$ and 3.0 respectively, $p<0.05)$ ] In the MTL, this effect was also significant in the delta and in the high-gamma frequency ranges $(M H=3.72$ and 2.33, respectively, $p<0.05$ ). 
Table 2. Load effects in regions of interest

\begin{tabular}{|c|c|c|c|c|c|c|c|c|c|c|}
\hline \multirow[b]{2}{*}{ Brodmann area } & \multicolumn{5}{|l|}{ Left } & \multicolumn{5}{|l|}{ Right } \\
\hline & $\begin{array}{l}\text { Delta } \\
(2-4 \mathrm{~Hz})\end{array}$ & $\begin{array}{l}\text { Theta } \\
(4-9 \mathrm{~Hz})\end{array}$ & $\begin{array}{l}\text { Alpha } \\
(9-14 \mathrm{~Hz})\end{array}$ & $\begin{array}{l}\text { Beta } \\
(14-28 \mathrm{~Hz})\end{array}$ & $\begin{array}{l}\text { Gamma } \\
(28-128 \mathrm{~Hz})\end{array}$ & $\begin{array}{l}\text { Delta } \\
(2-4 \mathrm{~Hz})\end{array}$ & $\begin{array}{l}\text { Theta } \\
(4-9 \mathrm{~Hz})\end{array}$ & $\begin{array}{l}\text { Alpha } \\
(9-14 \mathrm{~Hz})\end{array}$ & $\begin{array}{l}\text { Beta } \\
(14-28 \mathrm{~Hz})\end{array}$ & $\begin{array}{l}\text { Gamma } \\
(28-128 \mathrm{~Hz})\end{array}$ \\
\hline DLPFC & & & & & $\mathrm{L}+{ }^{1} \mathrm{~F}+{ }^{1,2,3}$ & & & & $\mathrm{~F}-$ & $\mathrm{L}+{ }^{1}$ \\
\hline $\mathrm{TC}$ & & $\mathrm{F}-$ & $\mathrm{F}-$ & & $\mathrm{F}+2,3$ & $\mathrm{~L}+\mathrm{F}-$ & & & $\mathrm{F}-$ & $\mathrm{F}+{ }^{2}$ \\
\hline MTL & & & & $\mathrm{F}-$ & $\mathrm{F}+{ }^{1,2,3}$ & $\mathrm{~L}+$ & & & $\mathrm{F}-$ & \\
\hline Hippocampus & & & & & $\mathrm{L}+{ }^{2} \mathrm{~F}+{ }^{2,3}$ & & $\mathrm{~L}+$ & & & $\mathrm{F}+{ }^{2,3}$ \\
\hline
\end{tabular}

For every combination of frequency (columns), hemisphere (columns), and brain region (rows), significant effects are indicated with $\mathrm{F}$ (face) or L (letter). Significant increases of oscillatory power with memory load for faces are indicated with $\mathrm{F}+$, and decreases with $\mathrm{F}-$. For letters, the coding is $\mathrm{L}+$ and $\mathrm{L}-$. The superscript numbers in the gamma columns indicate the sub-bands of gamma as follows: 1 , low ( $28-48 \mathrm{~Hz}) ; 2$, medium (48-90 $\mathrm{Hz}) ;$ or 3 , high $(90-100 \mathrm{~Hz})$. The regions of interest are the DLPFC, the TC, the MTL, and the hippocampus.

Figure 3 shows the locations of all cortical electrodes (not only those in the ROIs) that exhibited significant positive and negative load effects in the mid-gamma band.

Whereas positive load effects exceeded negative load effects primarily in the gamma frequency bands, at lower frequencies we observed several ROIs in which negative load effects were the dominant pattern. In the TC, the proportion of negative load effects exceeded that of positive load effects in the theta, alpha, and beta frequency bands $(M H=5.26,2.68,2.21$; all $p<0.05)$. In the MTL and DLPFC we also observed negative load effects that were dominant in the alpha and beta frequency bands, respectively $(M H=2.34$ and 2.89; $p<0.01)$.

We next asked whether faces and letters differed in the proportion of electrodes exhibiting positive gamma memory load effects. We found that the proportion of electrodes exhibiting positive gamma memory load effects was significantly larger for faces than for letters in the right DLPFC, MTL, and hippocampus $(M H=5.26,2.68$, and 2.21, respectively; $p<0.01)$ and in the left TC $(M H<2.96 ; p<0.01)$. In lower frequency bands, we found that the proportions of electrodes exhibiting negative memory load effects were significantly larger for faces than for letters in bilateral DLPFC, left MTL, right TC, right MTL, and right hippocampus ( $M H>2.03 ; p<0.05$ for all comparisons).

Whereas the previous analyses were well suited for studying oscillatory effects at individual electrodes, it is important to establish the degree to which the observed patterns generalize across participants whose electrode distributions are highly variable due to clinical considerations. To do this, we first determined the aggregate memory load effect for each participant within an ROI and then statistically evaluated the consistency of these effects across participants (see Materials and Methods).

The aggregate ROI analysis revealed significant increases in gamma power with memory load in a number of regions, including the left and right hippocampus (see Table 2). This aggregate ROI analysis showed similar results to the single electrode analysis, with increases in oscillatory power with memory load in the gamma and delta bands, and decreases in oscillatory power with memory load in the theta, alpha, and beta frequency bands. Most importantly, the aggregate analysis reproduced the loaddependent increases in gamma power in the left hippocampus (for both letters and faces), the right hippocampus (for faces), and the left MTL (for faces).

If the positive gamma memory load effect, observed primarily in the human hippocampus, is related to a process involved in memory maintenance, then one would expect this effect to be sustained throughout the $3 \mathrm{~s}$ retention interval. To address this question, we conducted an ANOVA across the three successive $1 \mathrm{~s}$ intervals during the retention interval. Specifically, we compared the across-interval variance of the sum- $z$-statistic to the withininterval variance, assessing significance using a permutation test. In the hippocampus, we found no significant difference between intervals for either letters or for faces $(p>0.05)$. For faces, we observed significant increases across intervals in the left DLPFC and the right MTL, whereas in TC we observed significant decreases. Gamma memory load effects for letters did not differ significantly across intervals in any regions (the time courses for these effects are presented in supplemental online materials, available at www.jneurosci.org).

Whereas the previous analyses excluded electrodes identified as being near the seizure focus and those exhibiting interictal spikes, we also conducted our aggregate ROI analyses by more conservatively excluding all electrodes overlying brain regions that were ultimately resected. Even though this more stringent criterion significantly reduced our statistical power for observing hippocampal effects, we nonetheless observed a statistically significant increase in gamma power (using a FDR of 0.005) with memory load for both faces (bilaterally) and letters (in the left hippocampus).

\section{Discussion}

We demonstrated that in the human hippocampus, $48-90 \mathrm{~Hz}$ gamma oscillatory power increases with memory load during the maintenance interval of a working memory task. This gamma pattern was also observed in other attention- and memoryrelated regions. The gamma increase with memory load in the hippocampus tended to be stronger for difficult-to-verbalize faces than for letters and did not significantly vary over the course of the maintenance interval.

Early lesion studies in animals and humans (Sidman et al., 1968; Wickelgren, 1968; Cave and Squire, 1992; Mayes et al., 2002) suggested that short-term maintenance of stimuli does not require the hippocampus. Neuroimaging studies have demonstrated similar results (Zarahn et al., 2005). These and similar findings led to the dominant viewpoint that working memory is hippocampus independent, whereas episodic memory requires the hippocampus (Baddeley and Warrington, 1970; Squire et al., 1993; Mumby, 2001).

Recent studies, however, have begun to challenge the view that the hippocampus does not support working memory function. Both lesion and neuroimaging studies in humans have shown the involvement of the hippocampus during certain types of working memory tasks, especially when participants are asked to maintain lists of perceptually complex or trial-unique visual stimuli (Holdstock et al., 1995; Owen et al., 1997; Buffalo et al., 1998; Stern et al., 2001). In animal studies, hippocampal lesions impair working memory when stimuli are repeated across trials (Rawlins et al., 1993), and hippocampal gamma activity is modulated during working memory tasks (Montgomery and Buzsáki, 2007).

In a previous electrophysiological investigation of working memory for faces, Axmacher et al. (2007) did not observe statistically significant load-dependent hippocampal oscillatory acti- 
vation. In that study, each list contained a unique set of photographic images of faces. As such, a probe item could be recognized on the basis of its overall familiarity. In contrast, we used a relatively limited set of abstract faces such that a given face would appear on multiple different lists. To successfully distinguish target and lure faces, our participants were required to focus their retrieval on the target list, effectively filtering out memories of items encoded on previous lists. As such, our task is likely to have involved far greater levels of interference from items learned on prior lists (i.e., proactive interference).

Insofar as working memory tasks require the successful targeting of memories based on their recency, these tasks rely on an essential function of episodic memory. Several computational models of episodic memory assume that items become associated with an evolving representation of temporal context, and that episodic memory arises when retrieval of an item evokes its previously learned contextual state (for review, Polyn and Kahana, 2008). The same contextual representation that marks memories as occurring in a given temporal context can also be used to discriminate among items studied in different contexts (Bower, 1972; Dennis and Humphreys, 2001; Howard and Kahana, 2002). In the case of the Sternberg task and other standard working memory tasks, the state of context that is active at the time of test will activate memories from the most recent target list, more so than memories from previous lists (Sederberg et al., 2008). If the process of linking item and context representations depends on hippocampal function (Paller and Wagner, 2002; Davachi, 2006; Polyn and Kahana, 2008), then hippocampal activation (as seen in gamma oscillations recorded in the local field potential) would be expected to increase with memory load, as we have observed here. This is consistent with findings that hippocampal gamma oscillations increase during successful memory formation and retrieval (Fell et al., 2001; Sederberg et al., 2007a). Hippocampal activation would also be expected to increase when experimental conditions make it more difficult to distinguish between items presented on the target list and items presented on earlier lists, as would occur in cases of lengthy retention intervals or retention intervals filled with distracting activity (Zola-Morgan et al., 1989; Aggleton et al., 1992).

In conclusion, our finding that hippocampal gamma oscillations increase with the number of faces or letters being maintained in working memory supports the emerging view that the human hippocampus supports a broad range of memory processes, including those involved in working memory maintenance. The present report also adds to an emerging body of evidence implicating gamma-frequency oscillations not only in attention and object recognition but also in higher order processes such as memory, learning, and the maintenance of goal states (Bauer et al., 2007; Sederberg et al., 2008; Jacobs et al., 2009).

\section{References}

Aggleton JP, Shaw C, Gaffan EA (1992) The performance of postencephalitic amnesic subjects on two behavioural tests of memory: concurrent discrimination learning and delayed matching-to-sample. Cortex 28:359-372.

Axmacher N, Mormann F, Fernandez G, Cohen MX, Elger CE, Fell J (2007) Sustained neural activity patterns during working memory in the human medial temporal lobe. J Neurosci 27:7807-7816.

Baddeley AD, Warrington EK (1970) Amnesia and the distinction between long- and short-term memory. J Verb Learn Verb Behav 9:176-189.

Bauer EP, Paz R, Paré D (2007) Gamma oscillations coordinate amygdalorhinal interactions during learning. J Neurosci 27:9369-9379.

Bower GH (1972) Stimulus-sampling theory of encoding variability. In:
Coding processes in human memory, Chap 5 (Melton AW, Martin E, eds), pp 85-121. New York: Wiley.

Buffalo EA, Reber PJ, Squire LR (1998) The human perirhinal cortex and recognition memory. Hippocampus 8:330-339.

Cave CB, Squire LR (1992) Intact verbal and nonverbal short-termmemory following damage to the human hippocampus. Hippcampus 2:151-164.

Curtis CE, D'Esposito M (2003) Persistent activity in the prefrontal cortex during working memory. Trends Cogn Sci 7:415-423.

Davachi L (2006) Item, context and relational episodic encoding in humans. Curr Opin Neurobiol 16:693-700.

Delorme A, Makeig S (2004) EEGLAB: an open source toolbox for analysis of single-trial EEG dynamics. J Neurosci Methods 134:9-21.

Dennis S, Humphreys MS (2001) A context noise model of episodic word recognition. Psychol Rev 108:452-478.

Fell J, Klaver P, Lehnertz K, Grunwald T, Schaller C, Elger CE, Fernandez G (2001) Human memory formation is accompanied by rhinal-hippocampal coupling and decoupling. Nat Neurosci 4:1259-1264.

Geller AS, Schleifer IK, Sederberg PB, Jacobs J, Kahana MJ (2007) PyEPL: a cross-platform experiment-programming library. Behav Res Methods 39:950-958.

Holdstock JS, Shaw C, Aggleton JP (1995) The performance of amnesic subjects on tests of delayed matching-to-sample and delayed matching-toposition. Neuropsychologia 33:1583-1596.

Howard MW, Kahana MJ (2002) A distributed representation of temporal context. J Math Psychol 46:269-299.

Howard MW, Rizzuto DS, Caplan JC, Madsen JR, Lisman J, AschenbrennerScheibe R, Schultze-Bonhage A, Kahana MJ (2003) Gamma oscillations correlate with working memory load in humans. Cereb Cortex 13:1369-1374.

Hwang G, Jacobs J, Geller A, Danker J, Sekuler R, Kahana MJ (2005) EEG correlates of verbal and nonverbal working memory. Behav Brain Funct $1: 20$.

Jacobs J, Hwang G, Curran T, Kahana MJ (2006) EEG oscillations and recognition memory: theta correlates of memory retrieval and decision making. Neuroimage 15:978-987.

Jacobs J, Korolev I, Caplan J, Ekstrom A, Litt B, Baltuch G, Fried I, SchulzeBonhage A, Madsen J, Kahana M (2009) Right-lateralized brain oscillations in human spatial navigation. J Cogn Neurosci. Advance online publication. Retrieved April 28, 2009. doi:101162/jocn.2009.21240.

Jensen O, Tesche CD (2002) Frontal theta activity in humans increases with memory load in a working memory task. Eur J Neurosci 15:1395-1399.

Kahana MJ (2006) The cognitive correlates of human brain oscillations. J Neurosci 26:1669-1672.

Loffler G, Yourganov G, Wilkinson F, Wilson HR (2005) fMRI evidence for the neural representation of faces. Nat Neurosci 8:1386-1390.

Mayes AR, Holdstock JS, Isaac CL, Hunkin NM, Roberts N (2002) Relative sparing of item recognition memory in a patient with adult-onset damage limited to the hippocampus. Hippcampus 12:325-340.

Meltzer JA, Zaveri HP, Goncharova II, Distasio MM, Papademetris X, Spencer SS, Spencer DD, Constable RT (2008) Effects of working memory load on oscillatory power in human intracranial EEG. Cereb Cortex 18:1843-1855.

Michels L, Moazami-Goudarzi M, Jeanmonod D, Sarnthein J (2008) EEG alpha distinguishes between cuneal and precuneal activation in working memory. Neuroimage 40:1296-1310.

Montgomery SM, Buzsáki G (2007) Gamma oscillations dynamically couple hippocampal CA3 and CA1 regions during memory task performance. Proc Natl Acad Sci U S A 104:14495-14500.

Mumby DG (2001) Perspectives on object-recognition memory following hippocampal damage: lessons from studies in rats. Behav Brain Res 127:159-181.

Owen AM, Morris RG, Sahakian BJ, Polkey CE, Robbins TW (1997) Double dissociations of memory and executive functions in working memory tasks following frontal lobe excisions, temporal lobe excisions or amygdalo-hippocampectomy in man. Brain 199:1597-1615.

Paller KA, Wagner AD (2002) Observing the transformation of experience into memory. Trends Cogn Sci 6:93-102.

Pantelis PC, van Vugt MK, Sekuler R, Wilson HR, Kahana MJ, (2008) Why are some people's names easier to learn than others? The effects of similarity on memory for face-name associations. Mem Cognit 36:1182-1195. 
Polyn SM, Kahana MJ (2008) Memory search and the neural representation of context. Trends Cogn Sci 12:24-30.

Postle B (2006) Working memory as an emergent property of the mind and brain. Neuroscience 139:23-38.

Ranganath C (2006) Working memory for visual objects: complementary roles of inferior temporal, medial temporal, and prefrontal cortex. Neuroscience 139:277-289.

Rawlins JNP, Lyford GL, Seferiades A, Deacon RMJ, Cassaday HJ (1993) Critical determinants of nonspatial working memory deficits in rats with conventional lesion of hippocampus or fornix. Behav Neurosci 107:420-433.

Robinson SE, Nagarajan SS, Mantle M, Gibbons V, Kirsch H (2004) Localization of interictal spikes using sam(g2) and dipole fit. Neurol Clin Neurophysiol 74.

Schiff SJ, Aldroubi A, Unser M, Sato S (1994) Fast wavelet transformation of EEG. Electroencephalogr Clin Neurophysiol 91:442-455.

Sederberg PB, Schulze-Bonhage A, Madsen JR, Bromfield EB, McCarthy DC, Brandt A, Tully MS, Kahana MJ (2007a) Hippocampal and neocortical gamma oscillations predict memory formation in humans. Cereb Cortex 17:1190-1196.

Sederberg PB, Schulze-Bonhage A, Madsen JR, Bromfield EB, Litt B, Brandt A, Kahana MJ (2007b) Gamma oscillations distinguish true from false memories. Psychol Sci 18:927-932.

Sederberg PB, Howard MW, Kahana MJ (2008) A context-based theory of recency and contiguity in free recall. Psychol Rev 115:893-912.

Sidman M, Stoddard LT, Mohr JP (1968) Some additional quantitative observations of immediate memory in a patient with bilateral hippocampal lesions. Neuropsychologia 6:245-254.
Squire LR, Knowlton B, Musen G (1993) The structure and organization of memory. Annu Rev Psychol 44:453-495.

Stern CE, Sherman SJ, Kirchhoff BA, Hasselmo ME (2001) Medial temporal lobe and prefrontal contributions to working memory tasks with novel and familiar stimuli. Hippocampus 11:337-346.

Sternberg S (1966) High-speed scanning in human memory. Science 153:652-654

Tulving E (1983) Elements of episodic memory. New York: Oxford UP.

van Vugt MK, Sederberg PB, Kahana MJ (2007) Comparison of spectral analysis methods for characterizing brain oscillations. J Neurosci Methods 162:49-63.

van Vugt MK, Sekuler R, Wilson HR, Kahana MJ (2009) Distinct electrophysiological correlates of proactive and similarity-based interference in visual working memory. Brain Res 1299:33-44.

Wickelgren WA (1968) Sparing of short-term memory in an amnesic patient-implications for strength theory of memory. Neuropsychologia 6:235-244.

Wilson HR, Loffler G, Wilkinson F (2002) Synthetic faces, face cubes, and the geometry of face space. Vision Res 42:2909-2923.

Zarahn E, Rakitin B, Abela D, Flynn J, Stern Y (2005) Positive evidence against human hippocampal involvement in working memory maintenance of familiar stimuli. Cereb Cortex 15:305-316.

Zola-Morgan S, Squire LR, Amaral DG (1989) Lesions of the amygdala that spare adjacent cortical regions do not impair memory or exacerbate the impairment following lesions of the hippocampus. J Neurosci 9:19221936. 